\title{
Continuum mechanical and computational aspects of material behavior
}

\author{
Eliot Fried* \& Morton E. Gurtin ${ }^{* *}$ \\ *Department of Theoretical and Applied Mechanics \\ University of Illinois at Urbana-Champaign \\ Urbana, IL 61801 \\ ${ }^{* *}$ Department of Mathematical Sciences \\ Carnegie Mellon University \\ Pittsburgh, PA 15213

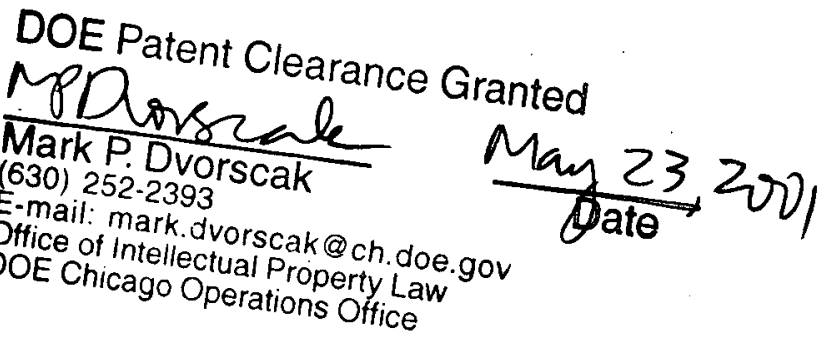




\section{DISCLAIMER}

This report was prepared as an account of work sponsored by an agency of the United States Government. Neither the United States Government nor any agency Thereof, nor any of their employees, makes any warranty, express or implied, or assumes any legal liability or responsibility for the accuracy, completeness, or usefulness of any information, apparatus, product, or process disclosed, or represents that its use would not infringe privately owned rights. Reference herein to any specific commercial product, process, or service by trade name, trademark, manufacturer, or otherwise does not necessarily constitute or imply its endorsement, recommendation, or favoring by the United States Government or any agency thereof. The views and opinions of authors expressed herein do not necessarily state or reflect those of the United States Government or any agency thereof. 


\section{DISCLAIMER}

Portions of this document may be illegible in electronic image products. Images are produced from the best available original document. 


\section{Summary}

The focus of this proposal is the application of continuum mechanics to materials science, specifically to the macroscopic characterization of material behavior at small length scales. The long term goals are: $(i)$ a continuum-mechanical framework for the study of materials that provides a basis for general theories and leads to boundary-value problems of physical relevance; and (ii) computational methods appropriate to these problems supplemented by physically meaningful regularizations to aid in their solution.

The following specific studies are planned:

- the development of a theory of polycrystalline plasticity that incorporates free energy associated with lattice mismatch between grains;

- the development of a theory of geometrically necessary dislocations within the context of finite-strain plasticity:

- the development of a gradient theory for single crystal plasticity with geometrically necessary dislocations;

- simulations of dynamical fracture using a theory that allows for the kinking and branching of cracks;

- computation of segregation and compaction in flowing granular materials.

We plan to continue our program of visits and research interaction with DOE laboratories. 


\section{Contents}

1 Results from prior DOE support 1

1.1 Defects . . . . . . . . . . . . . . . . . . 1

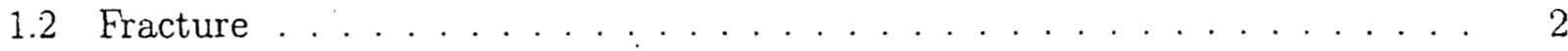

1.3 Phase transitions . . . . . . . . . . . . . . . . 2

1.4 Plasticity . . . . . . . . . . . . . . . . 4

1.5 Microforce theories for diffusion and recrystallization . . . . . . . . 5

1.6 Granular flow . . . . . . . . . . . . . . . . . . . 6

1.7 Papers arising from work under this grant $\ldots \ldots \ldots \ldots$

2. Interaction with DOE Laboratories 8

3 Description of proposed research 9

3.1 Plasticity . . . . . . . . . . . . . . . . . . . 9

3.2 Fracture . . . . . . . . . . . . . . . . . . . . . . 12

3.3 Granular flow . . . . . . . . . . . . . . . . . . . 13

4 Affiliations of collaborators 15

$\begin{array}{lr}\text { References } & 16\end{array}$

$\begin{array}{lr}\text { Biographical Sketch: Eliot Fried } & 19\end{array}$

$\begin{array}{ll}\text { Budget } & 21\end{array}$ 


\section{Results from prior DOE support}

\subsection{Defects}

\section{Dislocations in solids}

Since its derivation in 1950, the Peach-Koehler force has been synonymous with the (configurational) force on a dislocation, even though its particular form is a consequence of the linearity of the elastic model upon which it is based. Cermelli \& GurTin [1] studied the kinetics of screw dislocations in finite antiplane shear, concentrating on the derivation of equations that describe the motion of the dislocation. A general theory for the initiation and kinetics of glide and cross slip (changes in glide direction) is developed. The theory arcounts for the crystalline structure of the material and results in kinetic relations that allow for a critical resolved shear stress (Peierls force) and for the requirement that glide be restricted to slip planes. Equations are developed for the evolution of a system of dislocations in a linear elastic material, equations that should be amenable to analysis as well as to numerical simulation. It is shown that the notion of a phase portrait for a system of ODE s has a natural analog describing all possible trajectories of a dislocation. This notion allows for a discussion of phenomena such as cross-slip and fine cross-slip, in which a dislocation traverses a "wrinkled trajectory" composed of infinitesimal segments of glide in one direction interspersed with infinitesimal segments in a second direction. We believe this to be the first theoretical treatment of fine cross-slip.

\section{Point defects in liquid crystals}

The Oseen-Zöcher-Frank theory has allowed for the development of a comprehensive understanding of equilibria involving static point and line defects in nematic fluids. ${ }^{1}$ However, despite existent dynamical generalizations of this theory, the most standard of which is the Ericksen-Leslie theory, ${ }^{2}$ a comparable grasp of processes involving propagating defects is as yet undeveloped. Indeed, while ERICKSEN [6] has expressed confidence that the scope of the Ericksen-Leslie theory is adequate to describe dynamical processes in point defective nematic fluids, he has also remarked that little analytical progress has occurred on the basis of the theory. Using the framework of configurational forces developed in $[7,8]$, CERMELLI \& FRIED [9] focus on determining the full set of evolution equations describing defect propagation in nematic fluids. In addition to the conventional balances for deformational momentum, orientational momentum, and angular momentum, a configurational momentum balance is imposed; this balance is of trivial consequence away from any defect, but when localized at a point defect yields an independent evolution equation that determines the velocity of the defect with respect to the fluid in terms of the configurational force associated with the

\footnotetext{
${ }^{1}$ Cf. Oseex [2], Zöcher [3], Frank [4], Ericksex [5].

${ }^{2}$ Cf., e.g., ERICKSEN [6].
} 
nematic orientation field.

\subsection{Fracture}

GURTIN \& PODIO-Glidugli [10] have developed a general theory for crack propagation. In this theory, curving and kinking are allowed under the assumption that the crack propagate in a direction that maximizes the rate at which it dissipates energy. GURTIN \& SHVARTSMAN [11] have developed a theory for the propagation of plane cracks of arbitrary shape in three dimensions. This work shows that the line tension of the tip curve impedes the propagation of small cracks.

In conjunction with these works, the proposers have been developing a phase-field regularization of crack-propagation problems. This regularization, meant to yield computational procedures that avoid explicitly tracking the crack tip, involves extreme difficulties, as it must capture: ( $i$ ) singularities at crack tips; (ii) surface structure of crack faces; and (iii) the complicated interaction between the crack faces and the tip. We have the initial stages of a theory with encouraging computational support, but the project is in its infancy. We have been hesitant to publish this work until we have some assurance of the validity and viability of the model. This study bears some relation to the problem - in computer vision - of locating sharp changes.

\subsection{Phase transitions}

\section{Diffusional phase transitions in deformable solids}

GURTIN \& VOORHEES [12] have developed interface conditions for the evolution of a fully faceted interface separating elastic phases; metastable crystallographic orientations as well as stable orientations are allowed. Elastic stress is found to affect the velocity of a facet through the average value of the normal component of the jump in configurational stress over the facet. This work is a precursor to the development of phase-field theories appropriate for computations.

A lengthy review of the sharp interface and phase-field models of coherent phase transitions and their correspondence is the subject of a paper by FrIED \& GURTIN [13]. This invited paper forms part of a volume of the Journal of Statistical Physics in honor of J. W. CAHN.

An account of the major contributions of materials scientists, continuum physicists, and

mathematicians on evolving phase interfaces over the latter half of the $20^{\text {th }}$ century is given by FRIED [14].

\section{Incoherency and temperature discontinuities}

Gurtin, Weissmëller \& Larché [15] have developed an equilibrium theory for the 
deformation of curved interfaces between solid phases. This collaboration is continued in [16], which develops a broad generalization of classical relations for mean stress and mean strain appropriate to the study of material microstructures, and which discusses the role of such relations in an experimental program.

Cermelli, GuRTin \& LEONI [17] have investigated the role of interfacial energy for problems involving an epitaxial layer on a rigid substrate. Using the calculus of variations, resulting microstructures were determined for a large class of interfacial energies; the qualitative features of these microstructures demonstrate a strong dependence on the smoothness and convexity of the energy. This work should provide insight in deciding appropriate incoherency energies for a large class of physical problems.

In the combustion of a solid propellant, the temperature field may vary sharply across a thin layer separating the propellant from gaseous products. When the thickness of this layer is small, it is common to neglect its thickness and treat it as a sharp interface. FRIED \& SHEN [18] determine conditions that hold across such an interface and illustrate the role that these conditions serve by analyzing a solidification problem in which the temperature field is allowed to jump across the phase interface.

\section{Phase-field based simulations of twinning and coarsening in solids}

FRIED [19] has performed numerical simulations based on initial-boundary-value problems for the partial differential equations

$$
\begin{gathered}
\ddot{u}=c^{2}\left(\Delta u-z^{\prime}(\varphi) \kappa \cdot \nabla \varphi\right), \\
\epsilon \dot{\varphi}=\epsilon \Delta \varphi-\epsilon^{-1} f^{\prime}(\varphi)+z^{\prime}(\varphi) \kappa \cdot\left(\nabla u-\frac{1}{2} \kappa\right)
\end{gathered}
$$

which model twinning between two of the possible four variants that a monatomic bodycentered cubic single-crystal may display in the $\left(\begin{array}{lll}1 & 1 & 2\end{array}\right)[111]$ twinning mode. Here $u$ denotes the displacement along the [111] crystallographic axis; $\varphi$, the phase field, takes the value $\varphi=0$ in one variant and $\varphi=1$ in the other; $f$ is a symmetric double-well potential with wells at $\varphi=0$ and $\varphi=1 ; z$ is a smooth increasing function with $z(\varphi) \equiv 0$ for $\varphi \leq 0$ and $z(\varphi) \equiv 1$ for $\varphi \geq 1 ; 0<\epsilon \ll 1$ is a capillary constant; $\kappa$ with $|\kappa|=1 / \sqrt{2}$ is the twinning shear; $c>0$ is the underlying shear-wave speed.

The code uses 4- and 8-node isoparametric elements, with linear and quadratic interpolating functions, respectively. The fields $u$ and $\varphi$ are determined simultaneously. The nonlinear system at each time step is solved iteratively with a quasi-Newton method. The linear system at each step in the quasi-Newton iteration is solved using as iterative biconjugate gradient method with preconditioning based on an incomplete $L U$-decomposition or with diagonal scaling. Since a fine mesh is required to resolve the layers separating twin variants and the code does not yet incorporate an adaptive mesh algorithm, a one-dimensional condensed storage technique is used to decrease memory usage. The basic idea is to assemble 
the stiffness matrix, which is sparse and banded but unsymmetric, into a one-dimensional array.

Various numerical experiments indicate that the algorithm is stable: convergent, and accurate. The code was tested using rarious shapes for an initial twin inclusion. Regardless of the initial shape, the system converged, after a short initial transient, to a twin inclusion with a lenticular shape that is qualitatively consistent with experimental observations. ${ }^{3}$

Described also in [19] are simulations based on the elastic system with Cahn-Hilliard diffusion modeled by the equations

$$
\begin{gathered}
\dot{\varphi}=M \Delta \mu, \\
\Delta u=z^{\prime}(\varphi) \kappa \cdot \nabla \varphi \\
\mu=\epsilon^{-1} f^{\prime}(\varphi)-\epsilon \Delta \varphi-z^{\prime}(\varphi) \kappa \cdot\left(\nabla u-\frac{1}{2} \kappa\right),
\end{gathered}
$$

which describes coarsening in the presence of a misfit shear between phases. Here, $M>0$ is the mobility and $f$ and $z$ are as above.

\subsection{Plasticity}

Motivated by discussions with scientists at Sandia Livermore, where there is a strong interest in plasticity and damage, we began a program in crystalline plasticity, building on the framework of microforces presented in FRIED \& GURTIN $[23,24]$ and on the theory of configurational forces developed in $[7,8]$.

\section{Crystal plasticity}

The standard theory of plasticity for single crystals was generalized by GURTIN [25] to include constitutive dependencies on plastic strain gradients. ${ }^{4}$ The resulting theory, which demonstrates the role of free energy and the use of microforces work-conjugate to slip, has resulting yield condition a consequence of the underlying microforce balance. This theory should be applicable to phenomena, such as the formation of shear bands, in which the plastic strain suffers large spatial variations across narrow layers. The nonlocality of the theory requires supplementary boundary conditions; these are provided in a natural manner by the presence of microtractions.

\footnotetext{
${ }^{3}$ Cf. Hull [20], Rosakis \& TSaI [21]. These results are consistent with those reported by Rosakis, Hou \& LEFLOCH [22], who employed a level-set formulation in which interfacial energy is introduced nimerically. Because this surface energy is small, RosakIS, HoU \& LEFLOCH [22] are able to model tip splitting - a process of microstructural refinement. By taking $\epsilon$ to be sufficiently small, we should obtain similar results.

${ }^{4}$ There is a large and growing literature on gradient theories of plasticity. The point of view here is closest to FLECK \& HUTCHINSON [26] and GRACh, LUSK \& BAMMANY [27]. A critical discussion of computational mechanics at the mesoscale level is given by XEEDLEMAN [28], who presents a comparison of gradient theories and discrete-dislocation plasticity.
} 


\section{Configurational forces in plasticity}

CERmelli. Fried \& SELLERS [29] explore the relationship between finite-strain plasticity and a model of a continuum with continuous distributions of dislocations. The latter yields a formulation of plasticity in which the flow rule is viewed as an independent balance equation related to the dislocation density and the configurational stress. When the energetic contribution of the dislocation density is negligible, our theory yields both the flow rule and the yield condition of rate-independent finite plasticity. This result suggests an interpretation of the flow rule as a balance equation associated with microstructural defects such as dislocations. demonstrating a relationship between plastic flow as described by the plastic velocity gradient and the configurational stress.

In a related work, Cermelli, Fried \& Sellers [30] find that the configurational stress coincides with the classical Eshelby relation relative to the intermediate configuration determined by the multiplicative elastic-plastic decomposition; in fact, this configurational stress serves as the "driving force" for plastic flow.

\subsection{Microforce theories for diffusion and recrystallization}

\section{Diffusion}

FRIED \& SELLERS [31] have developed a theory of solute transport in fluids. This theory rests on the introduction of a force balance for the solute ${ }^{5}$ distinct from the macroscopic momentum balance associated with the mixture. Under certain circumstances, this force balance yields a Fickian constitutive relation for the diffusive solute flux, and, in conjunction with the solute mass balance, provides a generalized Smoluchowski equation for the mass fraction. Our theory furnishes a systematic procedure for generalizing convection-diffusion models of solute transport, allowing for constitutive nonlinearities and coupling between convection and diffusion. The underlying ideas have been applied to develop a description of atomic diffusion in deformable solids [32].

FRIEd \& SelLers [33] have developed a continuum theory of fluids consisting of orientable permanent dipoles. This theory accounts for the influences of rotary diffusion and externally applied fields. The governing evolution equations generalize the equations arising in common statistical models for dipolar fluids, such as the Debye theory of rotary diffusion.

\section{Recrystallization}

GURTIN \& LUSK [34] have developed a continuum framework for recrystallization. The driving force is energy stored in dislocation substructures. A relation is obtained characterizing the efficiency with which dislocation substructure is eliminated by moving grain

\footnotetext{
${ }^{5}$ Special forms of which have been used for at least a century.
} 
boundaries. Using a system of microforce balances, the sharp interface theory is shown to have a phase-field regularization that obviates the need to track individual grain boundaries.

\subsection{Granular flow}

A granular medium is a collection of solid particles together with an interstitial fluid. Geophysical events such as landslides and avalanches and industrial processes such as mixing and separation, grinding and crushing, and blasting all involve such media. In FRIED \& GURTIN [35], we develop a continuum mechanical theory for compaction and segregationby-particle-type in flowing granular media. A key goal of this theory is to account for the microphysical mechanisms that drive compaction and segregation. While continuum theories have been developed for monodisperse granular media: to our knowledge no such approach been applied to the study of segregation and compaction during the flow of polydisperse media. The approach we use is based on the treatment of voids as a distinct constituent. When the mixture-velocity of the medium is ignored, this theory gives rise to a system of quasilinear hyperbolic conservation laws. When segregation is ignored, so that the focus is on compaction, this system is replaced by a scalar hyperbolic equation that closely resembles equations arising in descriptions of traffic flow.

\subsection{Papers arising from work under this grant}

The work outlined above has led to the following published papers:

- P. Cermeli \& M. E. Gurtin, The motion of screw dislocations in crystalline materials undergoing antiplane shear: glide, cross-slip. fine cross-slip, Archive for Rational Mechanics and Analysis (1999) 148 3-52.

- P. Cermelli, M. E. Gurtin \& G. Leoni, Energies for incoherent interfaces, Interfaces and Free Boundaries 1 (1999), 81-105.

- E. Fried, Introduction. Fifty years of research on evolving phase interfaces, in Fundamental Contributions to the Continuum Theory of Evolving Phase Interfaces (J. M. Ball, D. Kinderlehrer, P. Podio-Glidugli \& M. Slemrod, Editors), Springer-Verlag, Berlin, 1999.

- E. Fried \& M. E. GurTin, Coherent solid-state phase transitions with atomic diffusion: a thermo-mechanical treatment, Journal of Statistical Physics 95 (1999), 13611427.

- E. Fried \& A. Q. Shen, Generalization of the Stefan model to allow for both velocity and temperature jumps, Continuum Mechanics and Thermodynamics 11 (1999), 277296. 
- M. E. Gurtin \& P. Podio-Guidugli, Configurational forces and a constitutive theory for crack propagation that allows for curving and kinking, Journal of the Mechanics and Physics of Solids 46 (1998), 1343-1378.

- M. E. Gurtin \& M. Shvartsman, Configurational forces and the dynamics of planar cracks in three-dimensional bodies. Journal of Elasticity 48 (1997), 167-191.

- M. E. Gurtin, J. Weissmüller \& F. LARche, A general theory of curved deformable interfaces in solids at equilibrium, Philosophical Magazine A 75 (1998), 10931109.

- M. E. Gurtin \& P. W. Voorhees, On the effects of elastic stress on the motion of fully faceted interfaces, Acta Materiala 46 (1998), 2103-2112.

- M. E. GurTin \& M. T. Lusk, Sharp-interface and phase-field theories of recrystallization in the plane. Physica $D 130$ (1999), 133-154.

In addition, the following have been accepted for publication:

- E. Fried \& S. Sellers, Theory for atomic diffusion on fixed and deformable crystal lattices, Journal of Elasticity.

- E. Fried \& S. Sellers, Microforces and the theory of solute transport, Zeitschrift für angewandte Mathematik und Physik.

- M. E. GurTin, On a gradient theory of crystalline plasticity, Journal of the Mechanics and Physics of Solids.

The following paper has been submitted:

- E. Fried \& S. Sellers, The Debye theory of rotary diffusion: History, derivation, and generalizations, Archive for Rational Mechanics and Analysis.

The following are in preparation:

- P. Cermelli \& E. Fried, Evolution equations for point defective nematic fluids.

- P. Cermelli, E. Fried \& S. Sellers, Finite crystal plasticity based on a microstructural model for defects.

- P. Cermelli, E. Fried \& S. Sellers, Configurational stress, yield, and flow in rate-independent plasticity.

- E. FrIEd, Phase-field based simulations of twinning and coarsening in solids.

- E. Fried \& M. E. Gurtin, Segregation and compaction in flowing granular materials.

- E. Fried, M. E. Glrtin \& V. Korchagin, Phase-field theory of damage in solids.

- M. E. Gurtin, J. IVeissmüller \& F. Lakché, Mean stress and mean strain in microstructures. 


\section{Interaction with DOE Laboratories}

Gurtin visited the Materials and Engineering Sciences Center, Sandia Livermore, in February, 1998 and then again in October 1998. There he gave three seminars:

- Configurational force as a basic concept in continuum physics.

- Microforces and Ginzburg-Landau-Cahn-Hilliard dynamics with deformation.

- On a gradient theory of crystalline plasticity.

During these visits GURTiN had discussions with: M. HorSTEMEYER on the use of internalstate variables to model plasticity and damage; D. BAMMANN on the use of phase-fields to nodel continuous distributions of dislocations; J. HOYT on computational materials science; DAN MOSHER on experimental mechanics; V. PRANTIL on polycrystalline plasticity - specifically on generalizing the standard Taylor theory. As a result of these discussions a joint project with PRANTIL was initiated; a continuation of this project forms a portion of this proposal.

GurTiN attended the DOE/MICS meeting at Los Alamos National Laboratory in December, 1999. While there he had discussions with LANL scientists C. ANDERSON, R. BROWNING, and P. SWART.

FRIED visited LANL in March, 1998. There, he gave a presentation:

- Continuum Phase Transitions.

Discussed were the correspondence between phase-field and sharp-interface theories for solidstate phase transitions and applications to the mechanically-induced detonation of energetic materials. There FRIED had discussions with a number of LANL scientists, including J. B. BIDZL, F. ADESSIO, and P. SWART.

FRIED attended the DOE/AMS Workshop on Mathematical Aspects of Materials Science Modeling at Gatlinburg in April 1998. There, he gave a seminar:

- Phase-field theories for solid-solid phase transitions: asymptotics and computational results.

FRIED visited the Materials and Engineering Sciences Center, Sandia Livermore, in April, 1999. There, he gave a seminar

- A continuum-mechanical theory for nematic elastomers.

During this visit he had discussions with: D. BAMMANN concerning continuum models for damage and concerning the role of constraints in plasticity; M. HORSTEMEYER on the use of phase-fields to model damage and phase transitions in solids; V. PRA.NTIL on the use of director theories to model the evolution of texture in polycrystalline solids. 
A recent paper by GRACH, LUSK \& BAMMANN [27] is based on the framework of microforces (and a concomitant microforce balance) developed by Fried \& GurTin $[23,24]$.

Even before the inception of the original grant the proposers exhibited a strong.interaction with DOE laboratories: GURTIN had served as a consultant for SNLA and LANL for approximately seven years and has coauthored papers with several DOE laboratory scientists, including R. BRowning of LANL. Moreover, the studies of FRIED and GuRTiN $[23,24]$ formed a basis for work of LUSK, KRAUS, AND JOU [36] on the CRADA project, a project directed toward developing a computer code for predicting distortion and residual stress in automobile transmission gears and industrial bearings during heat treatment. (The CRADA project also involves Ford, General Motors, and Torrington.)

\section{Description of proposed research}

In general, we propose a continuation of the research described above; specifically, the following investigations are planned:

\subsection{Plasticity}

\section{Polycrystalline plasticity}

Models of crystal plasticity based on Taylor's hypothesis - requiring that each grain undergo the macroscopic deformation of the polycrystal - predict textures that are generally too sharp. With the goal of ameliorating this inconsistency, GuRTIN \& PRANTIL ${ }^{6}$ have been working toward a generalization of the Taylor theory that accounts for free energy associated with the reluctance of the individual grains to rotate one relative to the other. The initial investigation - formulated within a rigid-plastic framework and based on the principle of virtual rork in conjunction with a microforce balance - results in a yield condition modified by the presence of an energetic (internal) moment $m^{g}$ for each grain $g .{ }^{7}$ For the special case of plane strain, letting $\vartheta^{1}, \vartheta^{2}, \ldots$ denote the angles of orientation of the individual grains, the moment $m^{g}$ has the form

$$
m^{g}=\frac{\partial \Psi\left(\vartheta^{1}, \vartheta^{2}, \ldots\right)}{\partial \vartheta^{g}}
$$

with free energy $\Psi$ such that

$$
\sum_{\text {grains } g} m^{g}=0 .
$$

\footnotetext{
${ }^{6}$ Materials and Engineering Science Center, Sandia Livermore.

'Aside from this modification, the theory is more or less the same as more standard theories of crystalline plasticity (cf., e.g., Kalindindi, Bronkhorst \& A.ind [37] and Asaro [38]). Our theory does not involve strain gradients.
} 
The equation (1). a consequence of objectivity, ensures that the internal moments not affect the standard macroscopic moment-balance, thus obviating the need for couple stresses. PRANTIL is currently modifying an existing laboratory code to investigate this modified yield condition for various simple choices for $\Psi$; hopefully to determine the viability of the proposed theory. We believe that a choice with minimum in the undeformed polycrystalline state would result in softer textures.

Here - continuing this collaboration with PRANTIL - the goal is to develop these preliminary studies into a rational theory set within a fully three-dimensional framework and supported by computational studies.

\section{Geometrically necessary dislocations}

Modern treatments of finite plasticity are based on the decomposition $F=F^{e} F^{p}$ of the deformation gradient into elastic and plastic contributions. An important feature of this decomposition is that, while $F$ is compatible (the gradient of a deformation), $F^{e}$ and $F^{p}$ are generally incompatible, a property that may be related to the formation of dislocations in the underlying lattice configuration. ${ }^{8}$ Preliminary results of CERMELLI \& GURTIN show that the incompatibility of $F^{e}$ and that of $F^{p}$ are together characterized by a single tensor field $^{9} G$ - the geometric dislocation tensor - that represents the Burgers vector measured per unit area in the lattice configuration. But what is most important, $G$ may be expressed equally well in two distinct forms: (i) in terms of $F^{p}$ and the referential curl of $F^{p}$; (ii) in terms of $F^{e-1}$ and the spatial curl of $F^{e-1}$. The standard assumption $\operatorname{det} F^{p}=1$ is not imposed; the general theory therefore allows for the formation of voids and the interaction of voids with other defects.

For plane strain, lattice planes convect locally to smooth surfaces in the deformed configuration; but this is generally not true for three-dimensional deformations. Preliminary studies indicate that this "surface roughening" is due essentially to the formation of screw dislocations. We propose to investigate this further.

For plane strain the form of the tensor $G$ simplifies, even more so when, in addition, elastic strains are neglected, rendering $F^{e}=R^{e}$ - a rotation that may be identified with a rotation-angle $\vartheta^{e}$. We plan to investigate this important special case further; preliminary investigations indicate simple, explicit formulas for $G$ in terms of $\vartheta^{e}$ and $\operatorname{grad} \vartheta^{e}$, where "grad" denotes the gradient with respect to the deformed configuration. Such results would seem helpful to applications, as experimenters typically measure relative rotation-angles of the lattice. ${ }^{10}$

\footnotetext{
${ }^{8}$ I.e., the "intermediate configuration"; the collection of local configurations toward which $F^{p}$ - and from which $F^{e}$ - map.

${ }^{9}$ There us a large literature on theories of continuous distributions of dislocations, proposed in various forms by Kondo, NYe. Bilby, Bullough, Smith, Seeger, Kröner, Günther, and others. Cf., e.g., the expository articles of KRÖNER [39] and SEEGER [40].

${ }^{10} \mathrm{Cf}$. the experiments -- carried out at Lawrence Livermore - of SCHWARTz, STölKEN, King, CAMPBELL.
} 
Additionally, the plan is to:

- Develop evolution equations for $G$, in general and for the special case of a single crystal.

- Develop a counterpart of $G$ appropriate to grain boundaries and evolving phaseinterfaces that are microstructurally incoherent.

\section{Gradient theory of single crystals with geometrically necessary dislocations}

The gradient theory of single crystals developed by GuRTIN [25] is based on a microforce balance that plays the role of the yield condition. We here plan to specialize this theory which allows for constitutive dependencies on $F^{p}$ and its gradient - to situations in which these dependencies are intended solely to account for the presence of geometrically necessary dislocations. Our plan is to model distortions of the crystal lattice due to such dislocations through a dependence of the free energy on the geometric-dislocation tensor $\boldsymbol{G}$. Preliminary investigations of CERMELLI \& GURTIN - for plane strain, a rigid lattice, and a free energy of the form $\frac{1}{2} \mu|G|^{2}$ - result in the standard yield condition for each slip system $\alpha$ modified by the presence of an energetic "backshear"

$$
\tau_{\text {back }}^{\alpha}=\mu \bar{s}^{\alpha} \cdot \operatorname{div}\left(\left(\operatorname{grad} \vartheta^{e}\right) \otimes \bar{s}^{\alpha}\right)
$$

with $\bar{s}^{\alpha}$ the deformed $\alpha$-th slip direction. Interestingly, the leading term in $\tau_{\text {back }}^{\alpha}$ is $\mu$ times the second derivative of $\vartheta^{e}$ in the direction of $\alpha$-slip. This modification renders the yield conditions nonlocal; specifically, these conditions form a system of partial differential equations that must be solved together with the standard macroscopic force balance.

The nonlocality of the theory necessitates additional boundary conditions related to the formation of geometrically necessary dislocations at the boundary. The simplest form of such conditions would be those associated with a free boundary; there the microtraction should vanish, a condition that, under the foregoing simplified assumptions, has the form

$$
\left(\bar{s}^{\alpha} \cdot \operatorname{grad} \vartheta^{e}\right)\left(\bar{s}^{\alpha} \cdot n\right)=0
$$

with $n$ the normal to the deformed boundary, so that either the $\alpha$-th slip direction is perpendicular to the boundary or the derivative of the lattice angle with respect to the $\alpha$-th slip direction vanishes.

LaSSILA, SUN \& Ada.MS [41]. 


\subsection{Fracture}

Our phase-field description of dynamical fracture - based on our theory of microforces $[23,24]$ - leads to the evolution equations

$$
\begin{aligned}
& \varrho \ddot{u}=\operatorname{Div}\left(\frac{\partial \Psi(E, \varphi)}{\partial E}\right), \\
& 3 \dot{\hat{r}}=\alpha \Delta \varphi-\frac{\partial \Psi(E, \varphi)}{\partial \varphi} .
\end{aligned}
$$

Here $u$ is the displacement; $\varphi$ is the phase field, where $\varphi=0$ is associated with virgin material and $\varphi=1$ with fractured material; $\varrho$ is the density;

\section{$-$}

$$
E=\frac{1}{2}\left(\nabla u+\nabla u^{\top}\right)
$$

is the infinitesimal strain; $\Psi(E, \varphi)$ is the coarse-grained energy density: 3 is a kinetic modulus associated with the rate at which cracks grow (and which may depend on $\varphi$ and $\dot{\varphi}$ ); $\alpha$ is a small parameter that characterizes the surface energy of the crack faces and-more pragmatically - smooths transitions between $\varphi \approx 0$ and $\varphi \approx 1$.

In our original approach to this problem, we took $\Psi(E, \varphi)$ to be of the form

$$
\Psi(E, \varphi)=\frac{1}{2}(1-(1-\epsilon) \varphi) E \cdot C[E]+\epsilon^{-1} f(\varphi),
$$

with $C$ the elasticity tensor, $f(\varphi)$ a double-well potential with equal minima at $\varphi=0$ and $\varphi=1$, and $\epsilon \ll 1$. In numerical simulations of mode-III fracture based on this choice, we have found that cracks grow unrealistically - with substantial thickening accompanying the propagation of the crack tip. Subsequently, we have found that the tenciency for cracks to thicken can be reduced, but not completely arrested, by taking $f(\varphi)$ to be a Lennard-Jones type potential with minimum at $\varphi=0$. (This choice of energy yields a theory which may be applicable to materials - such as concrete - that suffer damage in more diffuse patterns.)

With the goal of mimimizing crack thickening, we propose to pursue a further modification, based on the energy

$$
\Psi(E, \varphi)=\frac{1}{2}(1-\varphi)^{2} \boldsymbol{E} \cdot \mathbf{C}[\boldsymbol{E}]+\frac{1}{2} \epsilon^{-1} \nu \varphi^{2}, \quad \nu>0,
$$

which, in distinction to our previous choices, yields a quadratic decrease of elastic properties with damage. In statics, the mode-III version of the equations that arise for this choice coincide with equations used for image segmentation. Using the theory of $\Gamma$-convergence, AMBROSIO \& TORTORELLI $[42,43]$ have determined the $\epsilon \rightarrow 0$ limit of the image-segrnentation model; this limit yields equations identical to those describing a static mode-III crack in a linearly elastic solid. ${ }^{11}$ With SONER, we plan to study the $\epsilon \rightarrow 0$ limit of the dynamical

\footnotetext{
${ }^{11}$ We thank A. Braides (International School for Advanced Studies, Trieste) for valuable discussions regarding this model.
} 
three-dimensional equations that arise for the proposed choice of $\Psi(E, \varphi)$, with the particular goal of establishing a supplemental kinetic relation for the crack-tip velocity as a function of the driving force

$$
\oint_{\text {tip }}\left(\frac{1}{2}\left(E \cdot \mathbf{C}[E]+\varrho|u|^{2}\right) 1-(\nabla u)^{T} \mathbf{C}[E]\right) n d s,
$$

where $\oint_{\text {tip }} \ldots d s$ denotes an integral over an infinitesimal disk surrounding the crack tip.

Preliminary computations show that, without adequate resolution of regions within which $\varphi \approx 1$, the driving force acting at crack tips is underestimated to the extent that crack growth is suppressed. To overcome this difficulty, we plan to modify our code to include adaptive mesh refinement.

\subsection{Granular flow}

Here, we plan to focus on developing a code, based on the theory developed in [35], for the simulation of segregation and compaction in flowing granular material. An understanding of segregation is likely to lead to significant advances in industrial mixing processes - of vital importance in the chemical, pharmaceutical, powder metallurgy, glass, ceramic: paint, food, and construction industries - which, at present, are limited by a reliance on empiricallybased heuristics. Similarly, an understanding of compaction should allow for improvements in technologies such as resonant shock compaction - which is used to manufacture highvalued construction materials from coal ash, crushed concrete, and other materials and to provide high capacity, volume reduction and stabilization of contaminated soil.

Numerically, we face numerous challenges. To convey these, we ignore inertia, which - as is usual in theories involving mixtures is highly technical - is beyond the scope of this proposal. Writing $\varphi_{k}$ for the volume fraction of the $k$-th particulate constituent $(k=$ $1,2, \ldots K), \varrho$ for the density of the medium, defined by

$$
\varrho=\sum_{k=1}^{K} m_{k} \varphi_{k},
$$

with $m_{k}$ being the density of a particle of type $k, v$ for the volume-weighted mixture velocity,

$$
D=\frac{1}{2}\left(\operatorname{grad} v+\operatorname{grad} v^{\top}\right)
$$

for the strain-rate, $p$ for the pressure, and $b$ the external body force per unit mass of the medium (assumed to not vary between particle types), the resulting equations of our theory consist of: constituent volume balances

$$
\frac{\partial \varphi_{k}}{\partial t}+v \cdot \operatorname{grad} \varphi_{k}=-\operatorname{div} \jmath_{k}
$$

with $\jmath_{k}$. the flux of constituent $k$, given by

$$
\jmath_{k}=M_{k}(\varphi,|D|, \operatorname{det} D) b, \quad \varphi=\left(\varphi_{1}, \varphi_{2}, \ldots, \varphi_{K}\right) ;
$$


a macroscopic force balance

$$
\sim \operatorname{div} S+\varrho b=\operatorname{grad} p
$$

with extra stress given by

$$
S=2 \eta(\varphi,|D|, \operatorname{det} D) D
$$

and, writing $\varphi_{\text {voids }}$ for the volume fraction of voids, the constraint equations

$$
\varphi_{\text {voids }}=1-\sum_{k=1}^{K} \varphi_{k}, \quad \operatorname{div} v=0 .
$$

Despite the second of these constraints, the density $\varrho=\sum_{k=1}^{K} m_{k} \varphi_{k}$ is not generally constant; hence, our theory embraces density variations that accompany segregation (or mixing) and compaction.

Appearing in these equations are particulate mobilities $M_{k}>0$ and a viscosity $\eta>0$. Generally; we expect these functions to depend sensitively on the values of the particulate volume fractions. For instance, the mobilities of small particles should be substantially greater than those of large particles. Further, the viscosity should be an increasing function of the overall density $\varrho .{ }^{12}$

To treat these equations numerically, it would seem reasonable to develop a code that, at time step $n$, first solves the quasilinear hyperbolic system for the particulate volume fractions $\varphi_{k}^{n}$ given particulate volume fractions $\varphi_{k}^{n-1}$ and the velocity $v^{n-1}$ from the previous time step, and next, given the current particulate volume fractions $\varphi_{k}^{n}$, solves the remaining equations to determine $v^{n}$ and $p^{n}$. For the hyperbolic portion of the problem, we plan to use a spatial discretization based on the weighted essentially nonoscillatory method of LIU, OsHER \& CHAN [45] and a temporal discretization based on the total variation diminishing RungeKutta method of GotTlieb \& Shu [46]. Due to the presence of shocks, determination of the velocity and pressure fields at each time step will generally require the sclution of a quasilinear elliptic system with coefficients that, due to the previously discussed variations of the viscosity, possess strong discontinuities. Further, we anticipate that interactions between convection and diffusion may generate spatiotemporal pattern formation at various scales. Despite an existing host of powerful numerical methods for solving elliptic systems, we will need to develop a code with accuracy sufficient to resolve shocks and other features associated with segregation and compaction.

In particular, our plans include studies of segregation and compaction of a granular material:

- between horizontally sheared plates (plane Couette conditions);

- in a container subject to horizontal and/or vertical vibration;

\footnotetext{
${ }^{12}$ Cf., e.g., HORVATH, JANOSI \& VELLA [44], whose experiments indicate an exponential increase of viscosity with density.
} 
- in a rotating drum:

- subjected to shock-induced loading.

In addition, we intend to apply our model to geophysical phenomena such as avalanches and debris flows.

\section{Affiliations of collaborators}

- Paolo Cermelli

Dipartimento di Matematica

Università di Torino

- Via Carlo Alberto 10, 10123 Torino, Italy

- Frances Larché

GDPC

Université Montpellier II

Montpellier, France

- Mark T. Lusk

Division of Engineering

Colorado School of Mines

Golden, CO 80401

- Vincent Prantil Materials and Engineering Science Center

Sandia National Laboratory

Livermore, CA 94551

- Paolo Podio-Glidugli

Università di Roma "Tor Vergata"

via della Ricerca Scientifica

00173 Roma, Italy.

- Shaun Sellers

School of Mathematics

University of East Anglia

Norwich NR4 7TJ. England

- Mikhail Shvartsman

Department of Mathematics

University of St. Thomas

St. Paul, MN 55105

- H. Meté Soner

Department of Operations Research and Financial Engineering

Princeton University

Princeton, NJ 08.544 
- Jorge Weismüller

Fachbereich Physik

Universität des Saarlandes

D-66041 Saarbrüken, Germany

The following graduate students took part in the project: V. KorCHAGIN and A. Q. ShFn, Department of Theoretical and Applied Mechanics, University of Illinois; A. TsIKAS, Department of Mathematics, Carnegie-Mellon.

\section{References}

[1] P. Cermelli \& M. E. Gurtin, The motion of screw dislocations in crystalline materials undergoing antiplane shear: glide, cross-slip, fine cross-slip, Archive for Rational . Vechanics and Analysis, forthcoming.

[2] W. C. Oseen, The theory of liquid crystals, Transactions of the Faraday Society 29 (1933), 883-899.

[3] H. ZOCHER, The effect of a magnetic field on the nematic state, Transactions of the Faraday Society $29(1933), 945-957$.

[4] F. C. Frank, On the theory of liquid crystals, Discussions of the Faraday Society 25 (1958), 19-28.

[5] J. L. ERICKSEn, Equilibrium theory of liquid crystals, Advances in Liquid Crystals 2 (1976), 233-289.

[6] J. L. ERICKSEN, Liquid crystals with variable degree of orientation, Archive for Rational Mechanics and Analysis 113 (1991) 97-120.

[7] M. E. Gurtin, The nature of configurational forces, Archive for Rational Mechanics and Analysis 131 (1995), 67-100.

[8] M. E. Gurtin Configurational Forces as Basic Concepts of Continuum Mechanics, Springer, New York, 1999.

[9] P. Cermelli \& E. Fried, Evolution equations for point defective nematic fluids, in preparation.

[10] M. E. Gurtin \& P. Podio-Guidugli, Configurational forces and a constitutive theory for crack propagation that allows for curving and kinking, Journal of the Mechanics and Physics of Solids 45 (1998), 1343-1378.

[11] M. E. Gurtin \& M. Shvartsiran, Configurational forces and the dynamics of planar cracks in three-dimensional bodies, Journal of Elasticity 48 (1997), 167-191.

[12] M. E. Gurtin \& P. W. VoorheEs, On the effects of elastic stress on the motion of fully faceted interfaces, Acta Materiala 46 (1998), 2103-2112.

[13] E. Fried \& M. E. GuRTIN, Coherent solid-state phase transitions with atomic diffusion: a thermomechanical treatment; Journal of Statistical Physics 95 (1999), 1361-1427.

[14] E. FRIED, Introduction. Fifty years of research on evolving phase interfaces. in Fundamental Contributions to the Continuum Theory of Evolving Phase Interfaces (J. M. BALL. D. Kinderlehrer, P. Podio-Guidugli \& M. Slemrod. Editors), Springer-Verlag, Berlin, 1999.

[15] M. E. Gurtin, J. Weissmüller \& F. LarchÉ, A general theory of curved deformable interfaces in solids at equilibrium, Philosophical Magazine A 75 (1998), 1093-1109.

[16] M. E. Gurtin, J. Weissmüller \& F. Larché, Mean stress and mean strain in microstructures, in preparation.

[17] P. Cermelli, M. E. Gurtin \& G. Leoni, Energies for incoherent interfaces, Interfaces and Free Boundaries 1 (1999), 81-105. 
[18] E. Fried \& A. Q. ShEN, Generalization of the Stefan model to allow for both velocity and temperature jumps, Continuum Mechanics and Thermodynamics 11 (1999), 277-296.

[19] E. FRIED, Phase-field based simulations of twinning and coarsening in solids, in preparation.

[20] D. HuLl, Growth of twins and associated dislocation phenomena, in: Deformation Tuinning (R. E. ReEd-Hill, J. P. Hirth \& H. C. Rogers. Editors), Gordon and Breach, New York, 1963.

[21] P. Rosakis \& H.-Y. Tsai, On the role of shear instability in the modelling of crystal twinning, Mechanics of Materials 17 (1994), 245-259.

[22] T. Y. HOU, P. Rosakis \& P. LEFLOCH. A level-set approach to the computation of twinning and phase transition dynamics, Journal of Computational Physics 150 (1999), 302-331.

[23] E. Fried \& M. E. GuRTin, Continuum theory of thermally induced phase transitions based on an order parameter, Physica D 68 (1993), 326-343.

[24] E. Fried \& M. E. Gurtin, Dynamic solid-solid transitions with phase characterized by an order parameter, Physica D 72 (1994), 287-308.

[25] M. E. GurTin, On a gradient theory of crystalline plasticity, Journal of the Mechanics and Physics of Solids, forthcoming.

[26] L. A. FLECK \& J. W. HuTCHINSON, A phenomenological theory for strain gradient effects in plasticity, Journal of the Mechanics and Physics of Solids 41 (1993), 1825-1857.

[27] G. M. Grach, M. T. Lusk \& D. J. Bamann, An energetic framework for dislocation production and inelastic deformations, preprint.

[28] A. Needleman, Computational mechanics at the mesoscale, Acta Materialia. Special Millennium Issue (2000).

[29] P. Cermelli, E. Fried \& S. Sellers, Finite crystal plasticity based on a microstructural model for defects, in preparation.

[30] P. Cermelli, E. Fried \& S. Sellers, Configurational stress, yield, and flow in rate-independent plasticity, in preparation.

[31] E. Fried \& S. SEllers, Microforces and the theory of solute transport, Zeitschrift für angewandte Mathematik und Physik, forthcoming.

[32] E. Fried \& S. Sellers, Theory for atomic diffusion on fixed and deformable crystal lattices, Journal of Elasticity, forthcoming.

[33] E. Fried \& S. SELlers, The Debye theory of rotary diffusion: History, derivation, and generalizations, Archive for Rational Mechanics and Analysis, submitted.

[34] M. E. GurTin \& M. T. Lusk, Sharp-interface and phase-field theories of recrystallization in the plane, Physica $D 130$ (1999), 133-154.

[35] E. Fried \& M. E. GurTin, Segregation and compaction in flowing granular materials, in preparation.

[36] M. LUsK, G. KRAUSS \& H.-J. Jou, A balance principle approach for modeling phase transformation kinetics, Journal de Physique IV 5 (1995), 279-284.

[37] S. R. Kalidindi, C. A. Bronkhorst \&- L. Anand, Crystallographic texture evolution in bulk deformation processing of FCC metals, Journal of the Mechanics and Physics of Solids 40 (1992), 537-569.

[38] R. J. Asaro, Micromechanics of crystals and polycrystals, Advances in Applied Mechanics 23 (1983), $1-115$.

[39] E. KRÖNER, Allgemeine Kontinuumstheorie der Versetzungen und Eigenspannungen, Archive for Rational Mechanics and Analysis 4 (1960), 273-334.

[40] A. SeEger, Recent advances in the theory of defects in crystals, Physica status solidi 1 (1961), 669-698. 
[41] A. J. Schwartz, J. S. Stölken, W. E. King, G. H. Campbell, D. H. Lassila, S. Sty \& B. L. ADA.IS, Analysis of compression behavior of a [011] Ta single crystal with orientation imaging microscopy and crystal plasticity, Rept. UCRL-JC-133402, Lawrence Livermore National Laboratory.

[42] L. Ambrosio \& V. M. TorTorelli, Approximation of functionals depending on jumps by eiliptic functionals via $\Gamma$-convergence, Communications on Pure and Applied Mathematics 43 (1990). 9991036.

[43] L. Ambrosio \& V. M. Tortorelli, On the approximation of functionals depending on jumps by quadratic, elliptic functionals, Bollettino della Unione Matematica Italiana B 6 (1992), 105-123.

[44] V. K. Horvath, I. M. Janosi \& P. J. Vella, Anomolous density dependence of static friction in sand, Physical Review E 54 (1996), 2005-2009.

[45] X. D. LIL: S. Osher \& T. ChaN, Weighted essentially nonoscillatory schemes, Journal of Computational Physics 115 (1994), 200-212.

[46] S. Gottlieb \& C.-W. Shu, Total variation diminishing Runge-Kutta schemes, Mathematics of Computation 67 (1998), 73-85. 


\section{Biographical Sketch: Morton E. Gurtin}

\section{Professional EXPERIENCE}

Structures Engineer. Douglas Aircraft 1955-56; General Electric, 1956-59

Consultant, Sandia National Laboratory, 1967-74; Los Alamos National Laboratory, 1978, 1980-87; many other industrial organizations

Assistant and Associate Professor, Brown University, 1962-1966

Professor of Mathematics, Carnegie Mellon, 1966-

Director, Center for Nonlinear Analysis, Carnegie Mellon, 1991-1993

Alumni Professor of Mathematics, Carnegie Mellon, 1992-

\section{HONORS}

National Defense Fellow, Brown University, 1959-61

Senior.Fulbright-Hays Research Fellow and Guggenheim Fellow, University of Pisa, 1974

Honorary Fellow, Mathematics Research Center, University of Wisconsin, 1981-90

Brittingham Professor, University of Wisconsin, 1987-88

Ordway Professor, University of Minnesota, 1990

Honorary Degree "Laurea Honoris Causa" in Civil Engineering, University of Rome, July, 1994

Distinguished Graduate School Alumnus Award, Brown University Graduation, May, 1995

Volume 127 (1994) of the Archive for Rational Mechanics and Analysis in honor of 60th birthday

Volume 39 (1995) of the Journal of Elasticity contained 60th birthday retrospective of career

\section{SELECTED PUBLICATIONS}

- Multiphase thermomechanics with interfacial structure. 1. Heat conduction and the capillary balance law, Arch. Rational Mech. Anal. 104, 195-221 (1988)

- Multiphase thermomechanics with interfacial structure. 2. Evolution of an isothermal interface (with S. Angenent), Arch. Rational Mech. Anal. 108, 323-391 (1989)

- Multiphase thermomechanics with interfacial structure. 3. Evolving phase boundaries in the presence of bulk deformation (with A. Struthers), Arch. Rational Mech. Anal. 112, 97-160 (1990)

- The continuum mechanics of coherent two-phase elastic solids with mass transport (with P. W. Voorhees) Proc. Royal Soc. Lond. A 440, 323-343 (1993)

- On the kinematics of incoherent phase transitions (with P. Cermelli), Acta Metall. 42, 3349-3359 (1994)

- The dynamics of solid-solid phase transitions 2. Incoherent interfaces (with P. Cermelli), Arch. Rational Mech. Anal. 127, 41-99 (1994).

- Dynamic solid-solid phase transitions with phase characterized by an order parameter (with E. Fried), Physica D, 72 287-308 (1994)

- The nature of configurational forces, Arch. Rational Mech. Anal., 131, 67-100 (1995)

- Generalized Ginzburg-Landau and Cahn-Hilliard equations based on a microforce balance, Physica $D$ 92, 178-192 (1996)

- Configurational forces and a constitutive theory for crack propagation that allows for curving and kinking (with P. Podio-Guidugli), J. Mech. Phys. Solids, 46, 1343-1378 (1998)

- Coherent solid-state phase transitions with atomic diffusion: a thermo-mechanical treatment (with E. Fried), J. Statist. Phys. 95, 1361-1427 (1999) 


\section{EDUCATION AND HUMAN RESOLRCES}

Thesis advisor of 22 Ph.D. students of whom:

- eleven are teaching at universities or colleges in the United States; two being department chairmen and one a provost; of the ten, one is Black and three are women;

- two are professional scientists in the United States;

- one is the CEO and Chairman of the Board of a major corporation;

- eight are teaching in foreign universities.

Collaborations - resulting in published papers - with over fifty different scientists.

Research has resulted in:

- an article on Elasticity for the Handbuch der Physik;

- a book, Introduction to Continuum Mechanics, used as a text by Mathematics and Engineering Departments and referred to in the research literature;

- a recent book, Thermomechanics of Evolving Phase Boundaries in the Plane, and one just finished, Configurational Forces as Basic Concepts of Continuum Physics, both applicable to graduate education. 